This manuscript has been accepted for publication in a Special Issue of

Advances in Life Course Research

entitled

Theoretical and Methodological Frontiers in Life Course Research

edited by Laura Bernardi, Johannes Huinink, and Richard Settersten.

Please cite this manuscript as follows:

Mund, M. \& Nestler, S. (in press). Beyond the cross-lagged panel model: Next-generation tools for analyzing interdependencies across the life course. Advances in Life Course Research.

Before citing this work, please make sure you are using the latest version by checking https://osf.io/sjph7/

Date of this Version: September 24, 2018 


\title{
Beyond the Cross-Lagged Panel Model: Next-Generation Statistical Tools for Analyzing Interdependencies Across the Life Course
}

\author{
Marcus Mund \\ Friedrich-Schiller-Universität Jena
}

\author{
Steffen Nestler \\ Universität Leipzig
}

For decades, researchers have employed the Cross-Lagged Panel Model (CLPM) to analyze the interactions and interdependencies of a wide variety of inner- or supra-individual variables across the life course. However, in the last years the CLPM has been criticized for its underlying assumptions and several alternative models have been proposed that allow to relax these assumptions. With the Random-Intercept CLPM, the Autoregressive Latent Trajectory Model with Structured Residuals, and the Dual Change Score Model, we describe three of the most prominent alternatives to the CLPM and provide an impression about how to interpret the results obtained with these models. To this end, we illustrate the use of the presented models with an empirical example on the interplay between self-esteem and relationship satisfaction. We provide $\mathrm{R}$ and Mplus scripts that might help life course researchers to use these novel and powerful alternatives to the CLPM in their own research.

Keywords: second-order interdependencies, cross-lagged panel model, latent difference score model, autoregressive latent trajectory model, modeling change

When investigating individual development across the life course, researchers need to take a multitude of interdependencies between innerindividual and supra-individual factors into account (Bernardi, Huinink, \& Settersten, 2018). Bernardi et al. (2018) distinguish three basic,

Marcus Mund, Institut für Psychologie, FriedrichSchiller-Universität Jena, Humboldtstraße 11, D-07743 Jena; Steffen Nestler, Institut für Psychologie, Universität Leipzig, Neumarkt 9-19, D-04109 Leipzig.

Correspondence concerning this article should be addressed to Marcus Mund, Friedrich-SchillerUniversität Jena, Institut für Psychologie, Humboldtstraße 11, D-07743 Jena, E-mail: marcus.mund@gmail.com or first-order interdependencies: First, the timerelated interdependence of individual development refers to the association between an individual's past and how it affects his or her future. Second, the interdependence between life domains refers to the extent circumstances in one domain (e.g., work context) interact with circumstances in other contexts (e.g., family). Third, the multilevel interdependence of the life course refers to the interactions between individuals' actions and (a) the life courses of the individuals surrounding them, (b) with supra-individual factors (e.g., societal context) as well as (c) with inner-individual attributes (e.g., personality traits, haemodynamic reactivity). Importantly, the interplay among these first-order interdependencies creates a set of second-order interdependencies when investigating, for example, 
the interplay between changes in two characteristics, such as whether improved self-esteem leads to improvements in relationship satisfaction.

The analysis of such second-order interdependencies is particularly challenging as it requires the repeated assessment of a variety of innerindividual, contextual, social, and-depending on the research question-supra-individual variables characterizing each person. Furthermore, sophisticated statistical models have to be employed allowing a researcher to relate the repeated measurements of the variables to each other. Among these models, the Cross-Lagged Panel Model (CLPM; e.g., Biesanz, 2012) is one of the most popular approaches to analyze the interactions and reciprocal influences between variables over time.

Despite its widespread use, the strict assumptions of the CLPM have raised concerns regarding whether it is in fact appropriate for examining contemporary questions in life course research (Allison, 2009; Berry \& Willoughby, 2017; Hamaker, Kuiper, \& Grasman, 2015; Hertzog \& Nesselroade, 2003). This controversy has stimulated the development of a number of alternative approaches providing life course researchers with a fully packed box of new statistical tools. As of yet, however, a comparative overview of these models has been lacking. Therefore, our goal is to provide a comprehensive overview of prominent alternatives to the CLPM. The present article is organized as follows: First, we recapitulate the classical CLPM. Thereafter, we introduce three alternatives to CLPM: the Random-Intercept CLPM (Hamaker et al., 2015), the Autoregressive Latent Trajectory Model with Structured Residuals (Curran, Howard, Bainter, Lane, \& McGinley, 2014), and the Dual Change Score Model (Grimm, An, McArdle, Zonderman, \& Resnick, 2012). We then continue by comparing the three models with each other and with the fixed effects regression model (Allison, 2009) and the longitudinal multilevel model (Hox, 2010; Snijders \& Bosker, 2012), two widely-applied models in life course research. Finally, we will use an empirical example on the interplay between self-esteem and relationship satisfaction to illustrate the described models.

Before we start with introducing the models, we note that the aim of the present article is to equip researchers with statistical tools that might be more appropriate than the classical CLPM. Although we intend to introduce all models to a broad, non-technically oriented readership, we assume that readers are familiar with structural equation models (for an introduction, see Kline, 2011) and latent difference score models (for introductions, see Grimm, Ram, \& Estabrook, 2017; McArdle, 2009). For more technically oriented readers, we provide a formal description of the presented models in the Appendix. Finally, in an accompanying project hosted on the Open Science Framework (https://osf.io/sjph7/), we provide demo datasets and exemplar analysis syntax for the R package lavaan (Rosseel, 2012) and the commercial software package Mplus (Muthén \& Muthén, 2017) in order to enable life course researchers to adopt these models to further investigate a plenitude of innovative and important new research questions.

\section{Cross-Lagged Panel Model}

The CLPM is the standard model to examine rank-order changes and time-lagged associations between two longitudinally assessed variables (see Figure 1 for a CLPM with four measurement waves). It provides two types of coefficients that are of particular interest to life course researchers. First, the autoregressive paths ( $a 1$ and $a 2$ in Figure 1) provide information on the rank-order stability of $x$ or $y$, respectively (i.e., the stability of inter-individual differences; Mund, Zimmermann, \& Neyer, 2018). Second, the cross-lagged paths ( $c 1$ and $c 2$ in Figure 1) denote to what extent the prior scores of one variable relate to subsequent scores of the other variable. As the autoregressive effects and the cross-lagged effects are estimated simultaneously, the cross-lagged effects are often interpreted in terms of residualized change (Biesanz, 2012; Hertzog \& Nesselroade, 2003). 


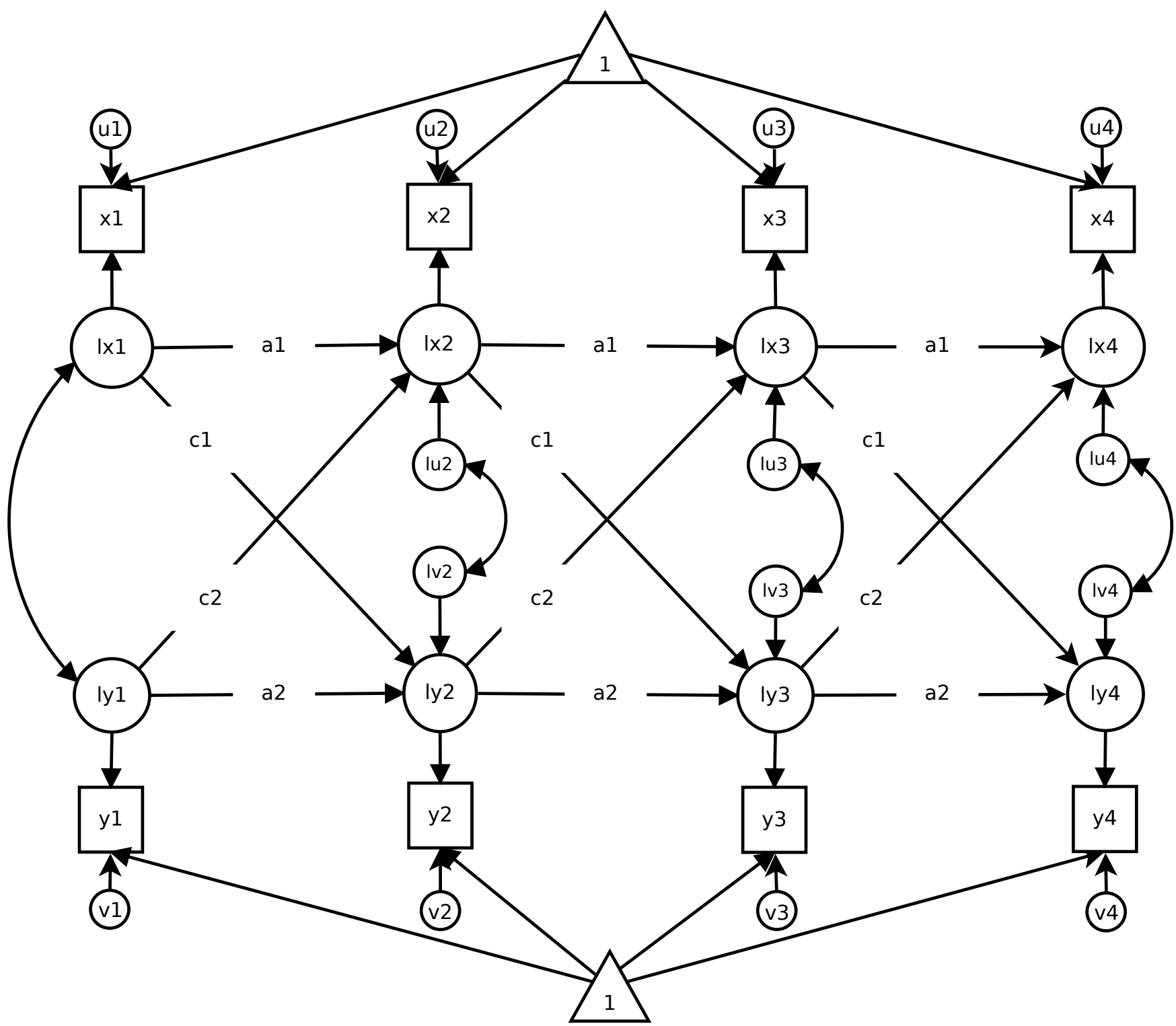

Figure 1. The Cross-Lagged Panel Model. exemplar model syntax are provided at https://osf.io/sjph7/. Squares represent observed variables (e.g., test scores), circles denote latent variables. Triangles represent intercepts. Single-headed arrows indicate regressions, double-headed arrows indicate correlations. The figure is available at https://osf.io/sjph7/ under a CC-BY 4.0 license.

Estimation of the CLPM requires that two variables had been assessed at two occasions at least. Furthermore, both the autoregressive and the cross-lagged paths may be constrained to be equal across time, although this is not a general precondition for the model to be applied. In fact, changes in the magnitude of either the autoregressive or the cross-lagged paths would usually be in- terpreted as shifts in the developmental system. Finally, the model can be extended to include multiple indicators of a construct and hence allows testing for measurement invariance (for more information, see Biesanz, 2012).

The CLPM is an interesting statistical model that can be employed to study many questions concerning individual development across the life 
course. However, several authors have pointed out that the CLPM makes a number of assumptions that might not be met in applied contexts or that researchers might not be aware of when using the model (Allison, 2009; Berry \& Willoughby, 2017; Hamaker et al., 2015).

First, the CLPM assumes that individuals fluctuate around a common group mean in each of the involved variables over time and, hence, that there are no stable between-person differences in these variables. Thus, the CLPM does not consider that the average level of a variable across time is higher for some individuals than for others. As a consequence, if stable between-person differences in $x$ or $y$ are present, they are included in the estimated autoregressive and cross-lagged paths (Berry \& Willoughby, 2017; Hamaker et al., 2015). The fact that the CLPM parameters may conflate withinperson and between-person effects increases the probability of false interpretations of the results. All three models that we will describe later in this article attempt to explicitly take trait-like betweenperson differences in the variables into account.

Second, the CLPM does not capture the mean structure of the involved variables. Hence, the model does not allow to examine whether there is mean-level change across time (such as in growth models; see Brüderl, Kratz, \& Bauer, 2018) and, related to the first point, whether there are between-person differences in this change beyond the autoregressive and cross-lagged effects. However, this is important as shifts in the rank-order of individuals in a variable are only one aspect of stability and change researchers might be interested in. Focusing on changes in the rank-order of individuals might disguise important developmental trends, since individuals might experience change that does not strongly affect the overall rank-order in the sample (Mund et al., 2018). For example, self-esteem has been found to increase on average between age 15 and 30 (Orth, Erol, \& Luciano, 2018) with the rank-order stability being as high as .60 in this age period (Trzesniewski, Donnellan, \& Robins, 2003). The Autoregressive La- tent Trajectory Model with Structured Residuals and the Dual Change Score Model attempt to take such between-person differences in within-person development into account.

Finally, the CLPM cannot be used to investigate whether the change between two consecutive measurement occasions is associated with further changes later in time. This hinders testing truly dynamic theories of life course development. At the same time, this also ignores the temporal interdependence of development stating that prior developments in one domain affect future developments in other domains (e.g., Bernardi et al., 2018). The Dual Change Score Model, in contrast, is capable of including changes in one domain as predictors of subsequent changes in the other domain.

In summary, the CLPM had a large impact on longitudinal life course research by influencing the conceptualization and investigation of developmental theories. However, the model makes a number of assumptions that may make its results difficult to interpret. Furthermore, it does not allow to represent the dynamic nature of life course theories. In the next section, we describe statistical models that address these limitations.

\section{Three Alternatives to the CLPM}

In the following, we describe the RandomIntercept CLPM (RI-CLPM), the Autoregressive Latent Trajectory Model with Structured Residuals (ALT-SR), and the Dual Change Score Model (DCSM). After having introduced these models, we will compare them to each other concerning some central aspects as well as to the multilevel growth model and the fixed effects regression model.

\section{Random-Intercept Cross-Lagged Panel Model}

The RI-CLPM as recently proposed by Hamaker et al. (2015) is displayed in Figure 2. It presents an alternative specification of the CLPM allowing to estimate the pure within-person autoregressive and cross-lagged effects. The basic idea behind the 
RI-CLPM is that, instead of all individuals varying around a common group mean over time, each individual fluctuates around his or her own rather stable, trait-like level over time with respect to the constructs under investigation. This assumption is implemented by defining a latent intercept factor for each construct across all time points. With regard to the empirical example that we will describe in detail later in this article, the intercept factor of relationship satisfaction, for instance, indicates that some individuals tend to be generally more satisfied with their relationships than other individuals.

Due to the separation of within- and betweenperson variation, the autoregressive effects provide information on the within-person stability in $x$ or $y$, respectively, instead of containing information on the (between-person) rank-order stability of these variables. Similarly, the cross-lagged effects pertain to within-person associations. Specifically, the $c 1$-path, for instance, denotes to what extent a deviation above or below the person-specific mean in $x$ (e.g., self-esteem) at an earlier point in time is associated with a subsequent deviation from the person-specific mean in $y$ (e.g., relationship satisfaction) controlling for previous deviations from the person-specific mean in this variable (Hamaker et al., 2015). Finally, the within-time correlations reflect within-person change associations and indicates to what extent deviations from the personspecific mean in $x$ (e.g., self-esteem) are accompanied by deviations from the person-specific mean in $y$ (e.g., relationship satisfaction).

Similar to the classical CLPM, the parameters of the RI-CLPM are estimated in structural equation modeling (SEM) software (see code at https: //osf.io/sjph7/). Estimating the model requires at least three measurements for each of the involved variables. Furthermore, the estimation of the RICLPM presupposes that the variables were measured without error, that is, the error variances of the observed indicators are constrained to be zero (for details and a further discussion, see Hamaker et al., 2015).
In summary, the RI-CLPM allows to separate within- from between-person effects. As it is also very flexible concerning the developmental mechanisms that it allows to examine, the RI-CLPM provides information on reciprocal associations between two (or more) variables within individuals.

\section{Autoregressive Latent Trajectory Model with Structured Residuals}

The ALT-SR is an extension of the Autoregressive Latent Trajectory Model (ALT), which was proposed as a combination of growth curve models and the CLPM (Bollen \& Zimmer, 2010; Curran \& Bollen, 2001). However, it turned out that the parameters of the ALT might be difficult to interpret (Voelkle, 2008). Furthermore, under certain conditions, the ALT is equivalent to a standard growth curve model with autoregressive relationships between the error terms (Hamaker, 2005; Jongerling \& Hamaker, 2011). The ALT-SR (also termed Latent Curve Model with Structured Residuals; Curran et al., 2014) addresses these two issues. Similar to the RI-CLPM, the model allows combining within- and between-person research questions. The bivariate ALT-SR for four measurement occasions is depicted in Figure 3.

As can be seen in Figure 3, the ALT-SR contains a latent growth curve part and a CLPM part. The growth curve part captures between-person differences in initial levels (the latent intercept factors in Figure 3) and development (the latent slope factors in Figure 3) of the variables of interest. Here, we used a linear growth model to illustrate the ALT-SR, but researchers are free to choose any time course in the growth curve (Brüderl et al., 2018; Ram \& Grimm, 2007). In fact, the adequacy of the parameter estimates relies on the correct specification of the growth curves for each of the involved variables (Voelkle, 2008). As in any other growth model (Brüderl et al., 2018; Grimm et al., 2017; Ram \& Grimm, 2007), the variance of the intercepts represent between-person differences in the variables under investigation at 


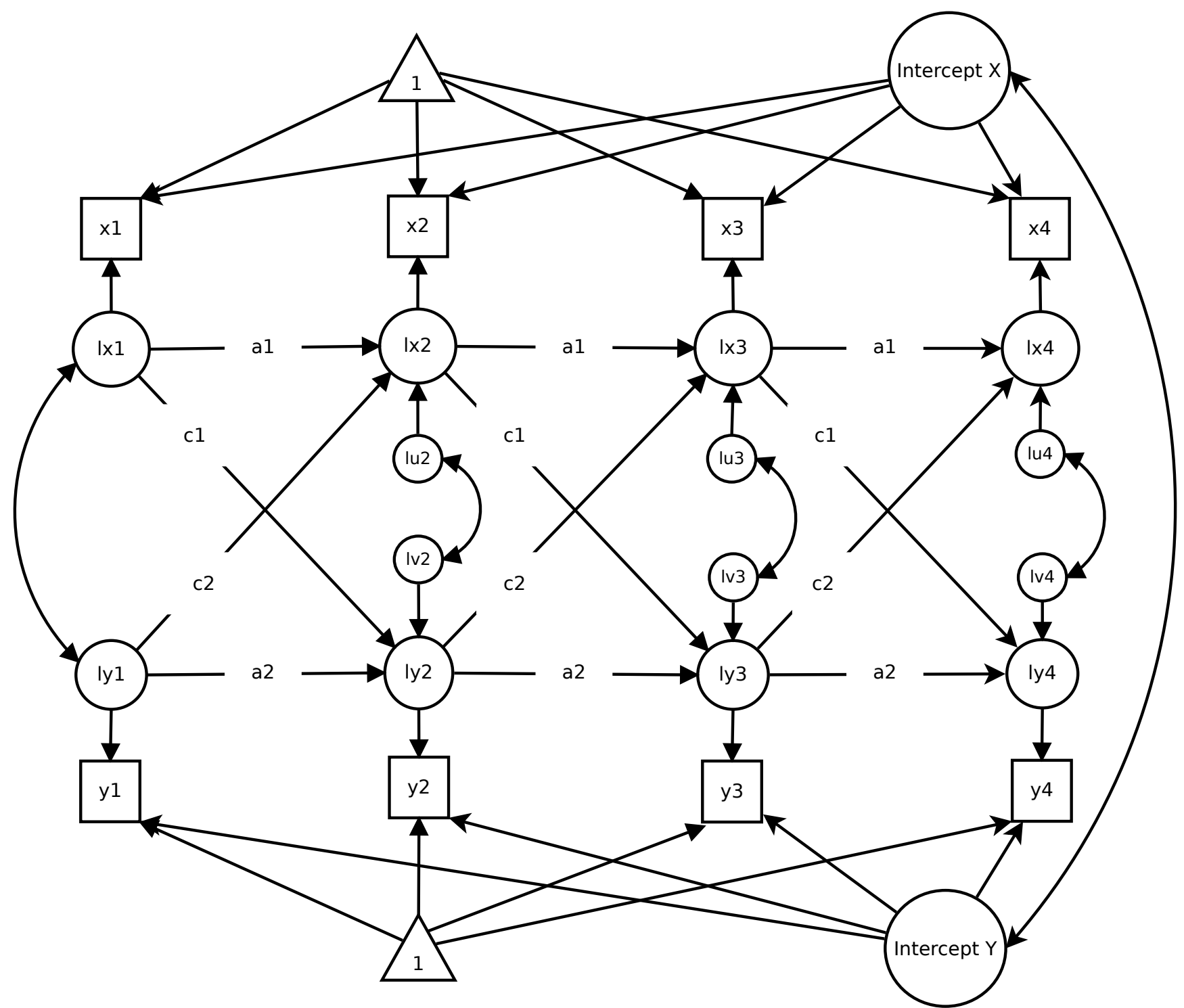

Figure 2. The Random-Intercept Cross-Lagged Panel Model. Details on implementation and exemplar model syntax are provided at https://osf.io/sjph7/, where the figure is also available under a CC-BY 4.0 license.

the first measurement occasion (e.g., some people are generally more satisfied with their relationships than others at the first time point). Accordingly, the mean and the variance of the slopes contain information on the average developmental trajectory over time (e.g., relationship satisfaction decreases on average) and between-person differences in this development (e.g., some individuals decrease more strongly than others regarding their relationship satisfaction), respectively. The corre- lation between the intercepts and the slopes can be interpreted as in standard multivariate growth models (Brüderl et al., 2018; Nestler, Grimm, \& Schönbrodt, 2015).

The CLPM part of the model is defined through the autoregressive and cross-lagged relationships between the residuals. This part of the model provides information on within-person dynamics as the residuals reflect time-point specific deviations from the person-specific mean and the person- 


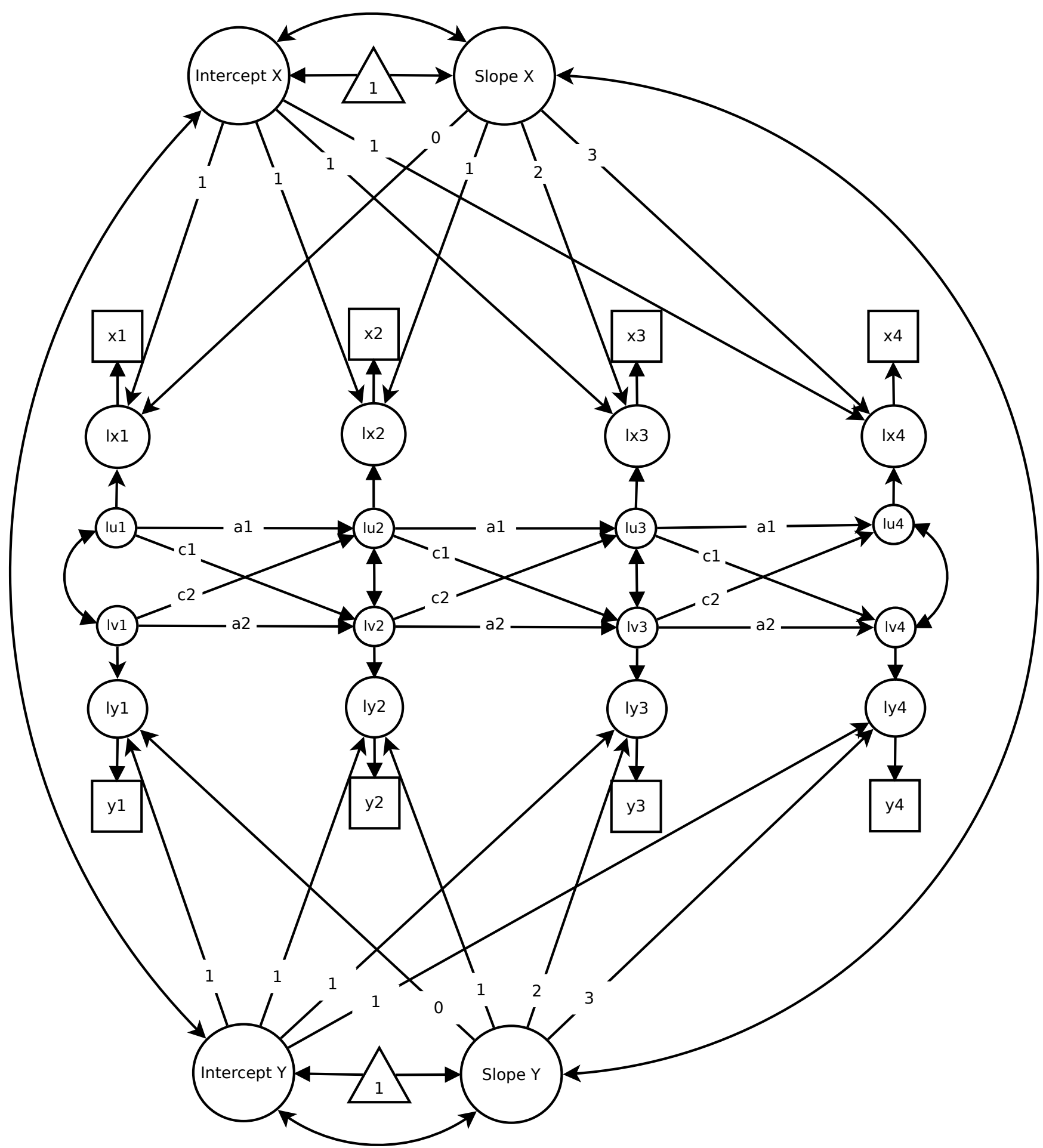

Figure 3. The Autoregressive Latent Trajectory Model with Structured Residuals. Details on implementation and exemplar model syntax are provided at https://osf.io/sjph7/, where the figure is also available under a CC-BY 4.0 license. 
specific growth curve. The autoregressive parameters describe the relationship between the withinperson residuals of the same variable at consecutive time points. The cross-lagged paths, by contrast, indicate whether within-person residuals of $x$ and $y$ at adjacent time points are related. Similar to the RI-CLPM, both parameters are interpreted relative to a person's developmental trajectory. Thus, a positive cross-lagged parameter would indicate that a person scoring higher than could be expected from her developmental trajectory on one variable tends to have values on the other variable that are higher than could be expected from the personspecific trajectory of this variable.

The ALT-SR is capable of accommodating many advanced features, which makes it an extraordinarily flexible and powerful statistical tool. For instance, it is possible to incorporate latent variables by setting up a second-order growth curve as the between-person component. Furthermore, the model can be extended by implementing predictors and consequences of between-person differences in the intercepts and slopes. Finally, it is also possible to investigate moderation by both categorical and continuous variables as well as mediation (Curran et al., 2014).

\section{Dual Change Score Model}

The Dual Change Score Model is part of the large family of latent difference score models (LDS), sometimes also termed latent change score models (LCS). Generally, an LDS combines features of both growth models and the CLPM (Hamagami \& McArdle, 2001; McArdle, 2009; McArdle \& Hamagami, 2001; Steyer, Eid, \& Schwenkmezger, 1997). The most basic form of an LDS for a single variable is depicted in Figure 4a. As can be seen in this figure, each time-specific latent score (e.g., lx2), except the first one, consists of the latent score of the previous time point (e.g., $l x 1)$ and a latent change score (e.g., $\Delta l x 1)$ reflecting the change from the prior time point to the subsequent time point. Thus, rather than investigating shifts in the rank-order between two time points as in the CLPM, latent difference score models examine the difference in a variable between adjacent measurement occasions (see also Castro-Schilo \& Grimm, 2018).

The LDS estimates the mean of a variable at the first time point, and the means of the difference variables at the subsequent time points. The model thus represents the initial mean and the meanlevel change at each of the subsequent time points across participants (i.e., the average of all individual changes over time; Mund et al., 2018). Furthermore, the variance of these variables can also be estimated. This allows researchers to examine between-person differences in the initial level and change.

A number of well-known growth models such as the intercept-only growth model or the linear growth model can be specified in the basic LDS framework (Grimm et al., 2017). The LDS in Figure $4 \mathrm{a}$, for example, would reduce to a linear growth model when the mean and the variance of all the latent difference variables were constrained to be equal. Another way to specify the latent growth model is to define another latent factor that loads on all latent difference variables. As it denotes change across all time points, the mean and the variance of this slope factor reflect a constant change component.

Further expanding this "slope"-LDS with timedependent effects (i.e., by specifying a regression between the latent scores at the prior time point and the latent change scores) results in one of the most well-known LDS models, namely the Dual Change Score Model (DCSM; see Figure 4b; Grimm et al., 2017; Hamagami \& McArdle, 2001; McArdle \& Hamagami, 2001). In the DCSM, overall change is decomposed into a constant change component and a proportional change component. In Figure 4b, SlopeX reflects the constant change component, which is similar to the slope factor in growth curves and captures the overall rate of change across all time points as well as between-person differences in this change. On the other hand, the proportional change component 
describes how change in a variable between adjacent measurement occasions depends on this variable's level at the prior time point.

The basic LDS and the DCSM depicted in Figures $4 \mathrm{a}$ and $4 \mathrm{~b}$ can be extended to the bivariate case so that the associations between two processes across time can be investigated. The bivariate DCSM is depicted in Figure 5. Adding timedependent effects between the latent change scores of the two variables (i.e., the gray paths in Figure 5) as suggested by Grimm et al. (2012) results in a more general version of the bivariate DCSM that we call extended bivariate DCSM. One of the key features that separates the extended bivariate DCSM from most other models is that it allows investigating the effects of changes in one variable on subsequent changes in the other variable (paths $d 1$ and $d 2$ in Figure 5), thereby enabling researchers to directly examine an assumption that is at the core of many theories of life course development (e.g., Bernardi et al., 2018).

In the bivariate DCSM, overall change is decomposed into a constant change component, a proportional change component due to the same variable, and a proportional change component due the other variable. The proportional change components indicates the extent to which constant change is limited or amplified by the same or the other variable's level at the prior time point. The correlation between the constant change scores can be interpreted just as the correlations between slopes in growth curve models.

Together with other models from the LDS framework, the DCSM is a powerful tool for investigating how the development of individuals across the life course is associated to both prior and future development in either the same or another domain. Thus, most of the contemporary theories of the life course positing temporal interdependence can be adequately modeled with the DCSM. Furthermore, latent variables, mediators, and moderators can all be implemented in the LDS framework. However, it should be noted that LDS in general do not separate within- and between-person variation, which might be unwanted in specific research contexts.

\section{Comparison of the Models}

So far, we have reviewed three alternatives to the classical CLPM that can be used to examine second-order interdependencies across the life course. In closing of this section, we highlight some of the similarities and differences between the models (see Table 1) and also compare them to the multilevel growth model and the fixed effects regression model.

Comparisons among the described models. As within- and between person variation are commingled in classical CLPM (Allison, 2009; Berry \& Willoughby, 2017; Hamaker et al., 2015), it is unclear which level of analysis the results of CLPM actually refer to. The RI-CLPM and the ALT-SR, in contrast, are quite clear with respect to their focal level: the RI-CLPM concentrates on within-person dynamics and captures betweenperson differences in the level of each variable by modeling the random intercepts (Hamaker et al., 2015). The ALT-SR is likewise capable of considering within- and between-person differences simultaneously (Bollen \& Zimmer, 2010; Curran \& Bollen, 2001; Curran et al., 2014). However, it extends the focus of the RI-CLPM by additionally considering between-person differences in withinperson development. The DCSM, as well as other models from the LDS family, does not separate within- and between-person effects so that the focal level of analysis is unclear (Grimm et al., 2012; McArdle, 2009).

Furthermore, whereas the CLPM and the RICLPM do not assume any general developmental trends, the ALT-SR allows modeling a wide variety of linear and nonlinear trajectories over time, depending on the number of measurement occasions. The DCSM, by contrast, yields an exponential trajectory whose type, however, depends on the constant growth component, the proportional component, and the average value at the first time point. Relatedly, both the ALT-SR and the DCSM 


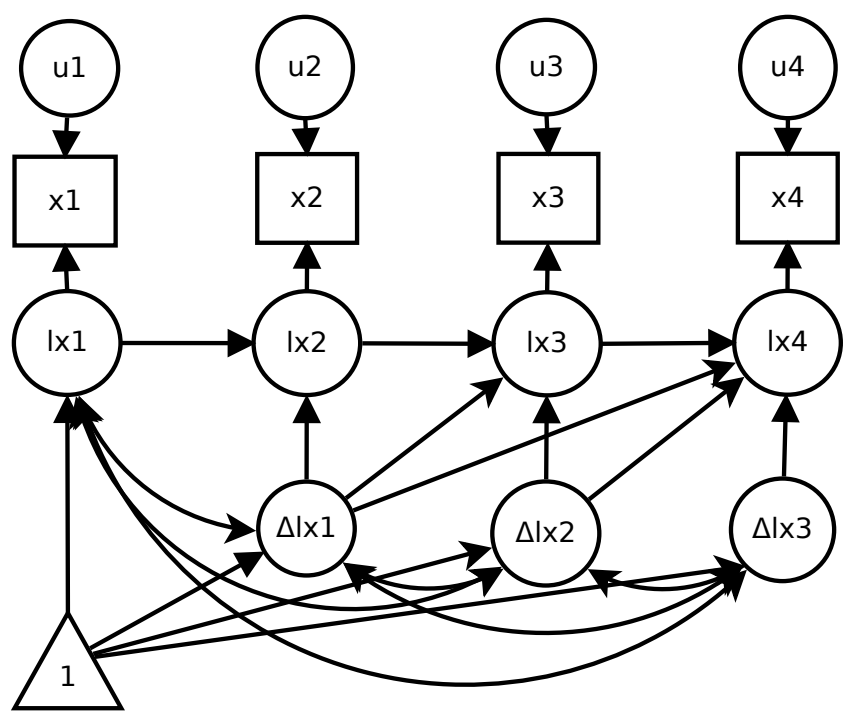

(a) Univariate LDS.

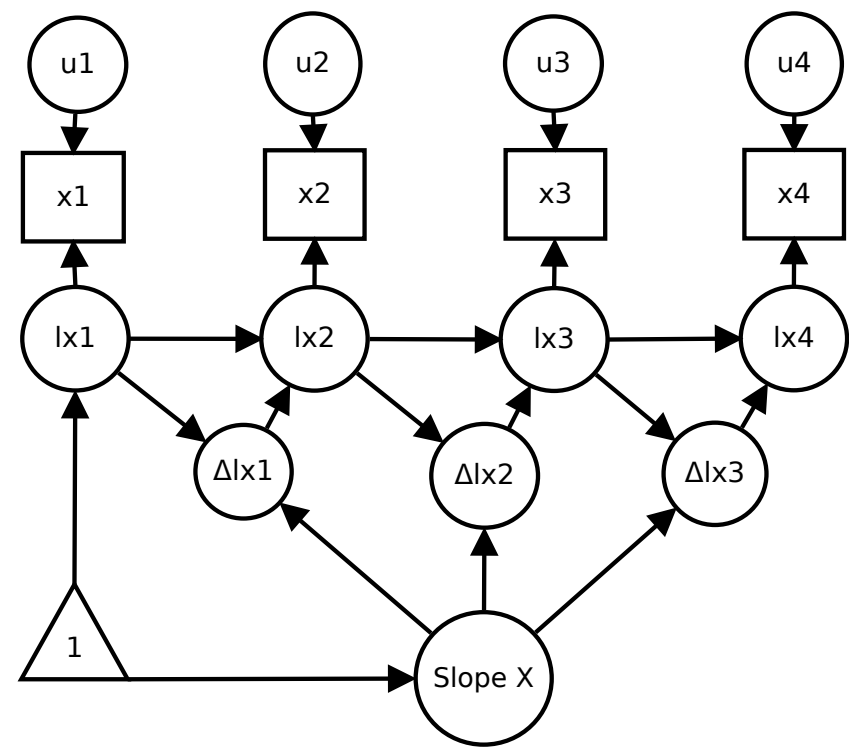

(b) Univariate DCSM.

Figure 4. The Latent Difference Score (Panel (a)) and the Dual Change Score Model (DCSM; Panel (b)). Details on implementation and exemplar model syntax are provided at https://osf.io/sjph7/, where the figures are also available under a CC-BY 4.0 license.

Table 1

Comparison of CLPM, RI-CLPM, ALT-SR, DCSM, RIRS, and FER

\begin{tabular}{|c|c|c|c|c|c|c|}
\hline Aspect & CLPM & RI-CLPM & ALT-SR & DCSM & $\operatorname{RIRS}^{a}$ & $\mathrm{FER}^{a}$ \\
\hline Level of Analysis & unclear & $\begin{array}{l}\text { within and } \\
\text { between }\end{array}$ & $\begin{array}{l}\text { within and } \\
\text { between }\end{array}$ & unclear & unclear & within \\
\hline $\begin{array}{l}\text { Differences in development } \\
\text { considered }\end{array}$ & no & no & yes & yes & yes & no \\
\hline $\begin{array}{l}\text { Model-implied } \\
\text { developmental trajectory }\end{array}$ & none & none & $\begin{array}{l}\text { linear and } \\
\text { non-linear }\end{array}$ & exponential & $\begin{array}{l}\text { linear and } \\
\text { non-linear }\end{array}$ & none \\
\hline Measurement Occasions & $\geq 2$ & $\geq 3$ & $\geq 4$ & $\geq 3$ & $\geq 2$ & $\geq 2$ \\
\hline
\end{tabular}

Note. CLPM: Cross-Lagged Panel Model; RI-CLPM: Random-Intercept Cross-Lagged Panel Model; DCSM: Dual Change Score Model; RIRS: Random-Intercept Random-Slope Model (Multilevel Growth Model); FER: Fixed Effects Regression Model. ${ }^{a}$ Note that the standard RIRS and FER do not include autoregressive or cross-lagged parameters.

but not the CLPM and the RI-CLPM allow considering between-person differences in change.

The presented models further differ with respect to the required number of measurement occasions. The CLPM is the least demanding and can be applied with data collected at two time points only. The RI-CLPM and the DCSM, by contrast, require at least three measurement occasions whereas the ALT-SR requires even four time points to be applied. A commonality of the presented models is that they require the measurement occasions to be equidistant for the parameter estimates to be meaningfully interpretable. In cases in which the measurement occasions are unevenly spaced for the entire sample or single individuals either by incident or by design, continuous time models should be considered (Voelkle \& Oud, 2013, 2015; Voelkle, Oud, Davidov, \& Schmidt, 2012).

Although all presented models have the potential to advance the understanding of individual development across the life course, they should not be encumbered with the hope of disentangling 


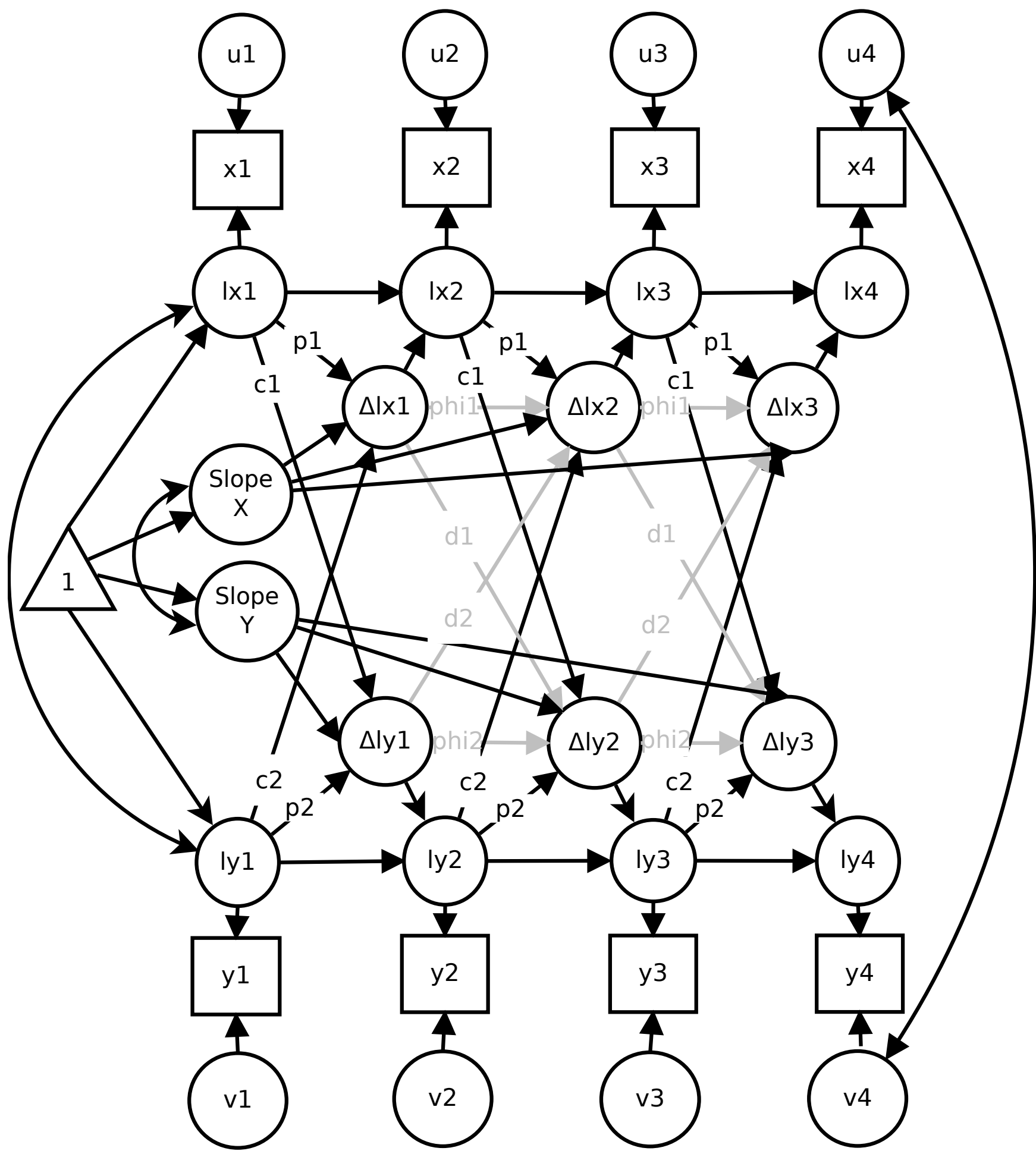

Figure 5. The bivariate DCSM with a recently proposed extension (gray paths). Details on implementation of this model and exemplar model syntax are provided at https://osf.io/sjph7/, where the figure is also available under a CC-BY 4.0 license.

causal mechanisms. Although the separation of within- and between-person variation as done, for 
instance, in the RI-CLPM and the ALT-SR can be seen as a major precondition for drawing causal inferences from panel data (for an alternative view, see VanderWeele, Hawkley, Thisted, \& Cacioppo, 2011), such inferences are valid only if all other conditions for causal interpretations are satisfied as well (Allison, 2009). As these assumptions are extremely strict, researchers should not expect to ever encounter a situation in which causal inferences from panel data are ultimately possible.

Comparison of the models with other established models. All models presented thus far are restricted versions of a structural equation model. The SEM framework is very prominent in life course psychology but less used by other life course social scientists. Here, multilevel models (Hox, 2010; Snijders \& Bosker, 2012) or linear fixed effects regression models (Allison, 2009) seem to be more prominent. This paragraph is therefore devoted to briefly compare the described models with these latter two models.

Multilevel models. With respect to multilevel models, we first note that a number of longitudinal multilevel models can be estimated in a SEM framework (e.g., Curran, 2003) and that the results are numerically identical to the results obtained using multilevel model software (but see Curran, Obeidat, \& Losardo, 2010, for exceptions). One of these multilevel models is the bivariate Random-Intercept Random-Slope model (RIRS) for longitudinal data in which a linear time variable is used to predict the longitudinal assessments of variables $x$ and $y$, respectively. Concerning the models introduced here, the RIRS represents a restricted version of the ALT-SR. Specifically, the linear ALT-SR model and the bivariate linear RIRS would yield the same results when the autoregressive and cross-lagged parameters are zero. When these two types of parameters are not zero, however, the bivariate RIRS separates the model-implied variance into between-person differences (i.e., in the intercepts and slopes) and error. The ALT-SR, by contrast, disentangles the variance into between-person differences, within- person differences across time (i.e., autoregressive and cross-lagged effects), and error.

As said, the RI-CLPM is a constrained version of the ALT-SR in the sense that no growth factor is incorporated into the model. Therefore, the RICLPM is similar to a bivariate random interceptonly model with the exception that autoregressive and cross-lagged effects are modeled for the residuals. Finally, the bivariate RIRS can also be considered a restricted version of the bivariate DCSM when the time-dependent effects on the change scores are zero and the variance terms of the latent change variables are set equal. In this case, the latent variable at the first time point reflects the intercept and the constant change factor reflects the slope factor in the RIRS. When these constraints are not made, the DCSM and the RIRS also differ in the model-implied variance (i.e., the DCSM separates the variance in between-person differences, error, and a mixture of between-person and withinperson effects).

Fixed effects regression model. In the fixed effects regression model (FER, see e.g., Allison, 2009), the relationship between time-varying predictors (e.g., self-esteem) and outcome variables (e.g., relationship satisfaction) is estimated while controlling for time-invariant person-specific effects. The latter is achieved by, for example, generating dummy variables for each person and by considering these dummy-variables together with the predictor variables in a linear regression model. The standard FER examines concurrent associations between the predictor variables and the outcome variables; the standard model thus does not provide estimates of autoregressive or crosslagged effects (but see Allison, 2009, for an extension of the FER involving lagged variables).

The main difference between the standard FER and the three introduced models is that the latter examine autoregressive and reciprocal effects while the former does not. Furthermore, the standard FER does not include a trend component which further differentiates it from the ALT-SR and the DCSM. Besides that, the RI-CLPM and the 
ALT-SR are similar to the FER in that both models include person-specific effects in the model (e.g., the intercept factor). All three models thus focus on the examination of within-person relationships.

\section{Empirical Illustration}

After having introduced the CLPM and three contemporary alternative approaches, we illustrate the interpretation of all models by an empirical example on the interplay between self-esteem (SE) and relationship satisfaction (RS). The reciprocal influences between these two variables have often been studied to investigate to what extent aspects of social relationships are influenced by and likewise further influence trait-like personality characteristics (e.g., Erol \& Orth, 2014; Mund, Finn, Hagemeyer, Zimmermann, \& Neyer, 2015; Orth, Robins, \& Widaman, 2012).

For the illustration, we use data from the first four measurement waves of the representative German Family Panel pairfam (Huinink et al., 2011). Pairfam is an ongoing study in which 12,402 individuals are interviewed yearly on a wide variety of demographic, socioeconomic, and personal issues (for more details, see http://www.pairfam.de/en). In our analyses, we only include the 2,665 individuals who provided data on both SE and RS. SE was measured using three items answered on a 5-point rating scale while RS was measured using one item with a 10-point rating scale (for more details, see Mund et al., 2015). Note that we use these data for illustrative purposes only; hence, we will not pay attention to specifics of the parameter estimates (e.g., whether paths should be freely estimated across time or not), or consider the effects of any covariates.

In the following, we discuss the key results of the analysis using the different models. The complete results, including the coefficients that we do not report in the tables, are available at https://osf. io/sjph7/.

\section{Cross-Lagged Panel Model}

The results of the CLPM are displayed in Table 2. The autoregressive paths amounted to 0.958 for SE ( $a 1)$ and 0.902 for RS ( $a 2)$, respectively, indicating that the rank-order of SE is slightly more stable than the rank-order of RS. Neither the path from SE to RS (c1), nor the reverse path (c2) reached statistical significance, which suggests no longitudinal association between SE and RS. The within-time correlations indicate a moderate codevelopment of both variables over time in a sense that stronger increases in SE are associated with stronger increases in RS, which must be assumed to be due to some third variable given the nonsignificant cross-lagged effects.

\section{Random-Intercept Cross-Lagged Panel Model}

We found positive autoregressive parameters when fitting a bivariate RI-CLPM to the data (see Table 2). As autoregressive and cross-lagged parameters reflect pure within-person estimates, this indicates that when an individual's SE (RS) is above his or her average level of SE (RS) at a specific time point, this individual is expected to score above his or her usual level of SE (RS) at the subsequent time point. The positive $c 1$-path in the example suggests that a score above the personspecific mean in SE at a given point in time is associated with a RS score above the person-specific mean in RS at a later point in time controlling for previous deviations from the person-specific mean in RS. The path from RS to SE (c2) was likewise positive but considerably smaller than the $c 1$-path (see Table 2).

The positive within-time correlations indicate that within-person increases in SE above the person's mean level are accompanied by personspecific increases in RS compared to the person's average (see Table 2). Notably, estimates of the correlations are considerably smaller than in the classical CLPM, because they only contain one source of variation (i.e., within-person). The traitlike between-person differences are captured by 
Table 2

Selected Results of the CLPM, RI-CLPM, ALT-SR, RIRS, and FER for the Interplay Between SelfEsteem and Relationship Satisfaction

\begin{tabular}{|c|c|c|c|c|c|c|c|c|c|c|}
\hline \multirow[b]{2}{*}{ Parameter } & \multicolumn{2}{|c|}{ CLPM } & \multicolumn{2}{|c|}{ RI-CLPM } & \multicolumn{2}{|c|}{ ALT-SR } & \multicolumn{2}{|c|}{$\operatorname{RIRS}^{a}$} & \multicolumn{2}{|c|}{$\operatorname{FER}^{a}$} \\
\hline & $b$ & S.E. & $b$ & S.E. & $b$ & S.E. & $b$ & S.E. & $b$ & S.E. \\
\hline$a 1$ & 0.958 & 0.021 & 0.194 & 0.019 & 0.113 & 0.028 & - & - & - & - \\
\hline$a 2$ & 0.902 & 0.041 & 0.115 & 0.019 & 0.083 & 0.031 & - & - & - & - \\
\hline$c 1$ & 0.015 & 0.052 & 0.231 & 0.048 & 0.173 & 0.065 & - & - & 0.282 & 0.035 \\
\hline$c 2$ & -0.013 & 0.008 & 0.024 & 0.005 & 0.021 & 0.007 & - & - & 0.030 & 0.004 \\
\hline$r_{I}$ & - & - & .355 & .033 & .349 & .090 & .345 & .039 & - & - \\
\hline$r_{S}$ & - & - & - & - & .058 & .701 & .284 & .133 & - & - \\
\hline$r_{l x 1, l y 1}$ & .396 & .037 & .039 & .026 & .051 & .046 & .083 & .014 & - & - \\
\hline$r_{l x 2, l y 2}$ & .562 & .081 & .159 & .015 & .145 & .021 & .083 & .014 & - & - \\
\hline$r_{l x 3, l y 3}$ & .562 & .081 & .141 & .013 & .136 & .020 & .083 & .014 & - & - \\
\hline$r_{l x 4, l y 4}$ & .562 & .081 & .147 & .013 & .149 & .022 & .083 & .014 & - & - \\
\hline
\end{tabular}

Note. CLPM: Cross-Lagged Panel Model; RI-CLPM: Random-Intercept Cross-Lagged Panel Model; RIRS: Random-Intercept Random-Slope Model (Multilevel Growth Model); FER: Fixed Effects Regression Model. $b$ : Unstandardized parameter estimate. $S . E$.: standard error; $N$ for all models is 2,665. Parameter estimates set in italics have a $p$-value $\geq .01$. $a 1$ and $c 1$ refer to autoregressive and lagged effects of self-esteem, respectively, while $a 2$ and $c 2$ refer to the same paths for relationship satisfaction. $r_{I}$ : Correlation between intercepts; $r_{S}$ : correlation between slopes. ${ }^{a}$ Note that the standard RIRS and FER do not contain autoregressive or cross-lagged paths.

the random intercepts, which are moderately correlated in our example. This shows that individuals with higher SE than other individuals tend to be more satisfied with their relationships than other individuals.

\section{Autoregressive Latent Trajectory Model with Structured Residuals}

As in the RI-CLPM, the trait-like betweenperson differences are captured by the random intercepts, while between-person differences in within-person development are captured by the slope factors. For SE, the mean of the linear slope factor was not significant while RS tended to decline $\left(\mathrm{M}_{\text {Slope } \mathrm{Y}}=-0.245, p<.001\right)$ over the four measurement occasions (see full results at https://osf.io/sjph7/). The correlation between the intercepts indicates that individuals with higher SE than other individuals at the first time point tend to be more satisfied with their relationships than other individuals. The correlation between the slope factors was not significantly different from zero indicating that no common development between SE and RS occur in our sample (see Table 2).

As in the RI-CLPM, the autoregressive and cross-lagged associations estimated with the ALTSR (see Table 2) pertain solely to within-person dynamics. Specifically, the positive autoregressive coefficients for SE $(a 1)$ and RS (a2) denote that higher-than-usual values of a person in a variable go along with higher-than-usual values in the same variable at the subsequent time point. The positive cross-lagged parameter $c 1$ indicates that increases in SE above the person-specific trajectory are predictive of above-average deviations in the personspecific development in RS. The estimate for $c 2$ is again positive and smaller than the estimate for $c 1$ and indicates that increases in RS above the person-specific development are predictive of more-than-usual increases in person-specific SE. 
Table 3

Selected Results of the Dual Change Score Models for the Interplay Between Self-Esteem and Relationship Satisfaction

\begin{tabular}{lcccrr}
\hline & \multicolumn{2}{c}{ DCSM } & & \multicolumn{2}{c}{ extended DCSM } \\
\cline { 2 - 3 } Parameter & $E S T$ & S.E. & & EST & S.E. \\
\hline Intercept X & 3.947 & 0.014 & & 3.944 & 0.014 \\
Intercept Y & 8.238 & 0.036 & & 8.281 & 0.038 \\
Slope X & 3.489 & 0.409 & & 3.623 & 0.582 \\
Slope Y & 0.120 & 3.334 & -2.012 & 4.335 \\
p1 & -1.369 & 0.139 & -1.601 & 0.172 \\
$p 2$ & 1.723 & 0.392 & & 2.017 & 0.556 \\
$c 1$ & -3.647 & 1.119 & -3.773 & 1.313 \\
$c 2$ & 0.232 & 0.055 & & 0.324 & 0.087 \\
phi 1 & - & - & 0.638 & 0.223 \\
phi2 & - & - & -2.450 & 0.465 \\
$d 1$ & - & - & 3.293 & 1.038 \\
$d 2$ & - & - & -0.343 & 0.124 \\
$r_{I}$ & .347 & .040 & .333 & .039 \\
$r_{S}$ & .783 & .108 & .770 & .120 \\
\hline
\end{tabular}

Note. EST: Point estimate. S.E.: standard error; $N$ for all models is 2,665. Parameter estimates set in italics have a $p$-value $>.01$. $r_{I}$ : Correlation between intercepts; $r_{S}$ : correlation between slopes.

The positive within-time correlations suggests that deviations from the person-specific SE above the person's mean level and developmental trajectory are accompanied by positive person-specific deviations in RS compared to the person's average and development.

\section{Dual Change Score Model}

Although the DCSM differs from the RI-CLPM and the ALT-SR in several ways (e.g., absence of autoregressive effects in DCSM), a common feature of the DCSM and the ALT-SR is the correlation between both intercepts and slopes. With regard to the intercepts, the positive correlation displayed in Table 3 indicates that individuals higher in SE than others tend to report higher RS than other individuals.

In order to interpret the association between SE and RS in our current example using the DCSM, it is necessary to consider all parameters estimated by the model: Starting from values of 3.947 (SE) and 8.238 (RS), the constant change components amounted to $3.489(S x)$ for $\mathrm{SE}$ and to 0.120 for RS $(S y)$, respectively. Estimates for the proportional change component were -1.369 for SE $(p 1)$ and 1.723 for RS ( $p 2)$, respectively. For the crosslagged effects, the bivariate DCSM yielded an estimate of -3.647 for the path from SE to RS $(c 1)$ and 0.232 for the reverse path $(c 2)$, respectively. For SE, this indicates that SE increases constantly in each time interval (i.e., positive constant change component), but that this change is pulled downwards the more time points are passed (i.e., negative proportional change) and accelerated by the earlier level of RS (i.e., positive cross-lagged effect). With regard to RS, the DCSM indicates a slight constant yet nonsignificant increase over time (i.e., positive constant change) that is even amplified the more time points are passed (i.e., positive proportional change component). Prior levels of SE were found to decelerate this change (i.e., negative cross-lagged path; see Supplemental Material at https://osf.io/sjph7/ for full equations on this example).

The results of the extended bivariate DCSM (right-hand column in Table 3) can be interpreted accordingly. However, this model also allows examining the effects of previous changes in one variable on subsequent changes in another variable. In terms of our example, we found that the effect of changes in SE on subsequent changes in RS (d1) amounted to 3.293 while the reverse effect $(d 2)$ amounted to -0.343 . Thus, while larger change in SE goes along with larger change in RS, the reverse is not the case. Furthermore, the paths linking changes in SE (phi1) and RS (phi2), respectively, over time amounted to 0.638 and -2.450 . This indicates that stronger changes in SE in the earlier interval are followed by stronger changes in SE in subsequent intervals, while stronger changes in RS are followed by weaker subsequent changes therein. 


\section{Random-Intercept Random-Slope Model}

As the RIRS does not contain autoregressive or cross-lagged paths, our comparison with the other models is restricted to the correlational associations between SE and RS. As in the RI-CLPM and the ALT-SR, between-person differences are captured by the intercept factors of the RIRS. As can be seen in Table 2, the correlation between RS and SE as estimated by the RIRS closely matches the correlations between the intercepts and slopes estimated in the RI-CLPM, the ALT-SR, and the DCSM, respectively, indicating that individuals higher in SE than others also report higher RS.

Similar to the ALT-SR, the mean of the linear slope factor was not significant for SE but indicated decreases in RS $\left(\mathrm{M}_{\text {Slope } \mathrm{Y}}=-0.249, p<\right.$ .001 ) over the four measurement occasions (see full results at https://osf.io/sjph7/). The correlation between the slopes, which was not significant in the ALT-SR, was significant in the RIRS. This indicates that changes in SE were associated with changes in RS over time (see Table 2). This difference to the ALT-SR is due to the substantial autoregressive and cross-lagged effects between SE and RS, which are not accounted for by the RIRS.

\section{Fixed Effects Regression}

Similar to the RIRS, the classical FER does not contain autoregressive or cross-lagged effects. To estimate reciprocal effects, it is necessary to run two separate models, one to estimate the regression of SE on RS and one for the reverse path (Allison, 2009). The estimated effects, however, pertain to pure within-person dynamics.

For the first path from SE on RS (c1), the results of the FER converge well with those obtained with the RI-CLPM and the ALT-SR (see Table 2). Specifically, the positive $c 1$-path indicates that deviations above the person-specific mean in SE at a given point in time are associated with deviations above the person-specific mean in RS. The reverse path from RS to SE (c2) that was estimated in a separate model, was likewise positive but consider- ably smaller than the $c 1$-path (see Table 2). It indicates that individuals who score above their mean in RS are expected to score above their personspecific mean in SE.

\section{Summary}

We demonstrated the functioning of the CLPM, RI-CLPM, ALT-SR, and DCSM by applying these models to an empirical example on the interplay between self-esteem and relationship satisfaction in a representative German sample. While the CLPM indicated no interplay between SE and RS, all other models indicated reciprocal relationships between these two variables. While the RI-CLPM and the ALT-SR separate within- from betweenperson variation and thus equip their cross-lagged parameters with a clear and unambiguous meaning, different sources of variation are conflated in the CLPM, rendering the interpretation of the cross-lagged parameters particularly difficult to interpret and likely biased. The RI-CLPM and the ALT-SR converged on virtually identical parameter estimates in those parts of the model shared by both analytical approaches (correlation of intercepts, cross-lagged and autoregressive effects, within-time correlations). Additionally, the ALTSR indicated a developmental trend over time at least for RS, providing a connection to the DCSM in which such developmental trends were also observed.

While the CLPM examines residualized change, the DCSM builds on difference scores (CastroSchilo \& Grimm, 2018). As a result, the DCSM stands out and is not readily comparable to the other models. However, it is evident from the example that the DCSM allows researchers to examine complex change trajectories and factors influencing it. Particularly the extended bivariate DCSM provides a highly dynamic perspective on the analysis of second-order interdependencies across the life course. It should be kept in mind, though, that unlike in the RI-CLPM and ALT-SR, within- and between-person variance are not separated. 
Concerning the widely-applied and wellestablished RIRS and FER, the results of our empirical example demonstrate that they are more specific than the other models discussed in this article. The results of the FER closely match the results obtained for the within-person parts of the RI-CLPM and the ALT-SR. However, the former examines concurrent associations between the involved variables while the latter investigate lagged relationships. The RIRS, in contrast, resembles the between-person parts of the RI-CLPM, the ALT-SR, and the DCSM. In this respect, results of RIRS converge with the other models regarding the correlation of the intercepts. Concerning the associations between the slopes, the results obtained with RIRS differ from those obtained with the ALT-SR, which is due to RIRS not incorporating autoregressive or cross-lagged effects. To summarize, our results show that the three approaches presented in this paper substantially extend the RIRS and the FER by providing additional information on the longitudinal interplay between two variables that might be of interest for life course researchers.

\section{Conclusion}

Trying to understand the life course of individuals is an ambitious endeavor that requires a tailored set of tools regarding study design, data collection, and data analysis (Bernardi et al., 2018). Across their life course, individuals navigate through and interact in different contexts. These interactions between two complex systems (individual and environment) as well as interactions within individuals create a set of interdependencies that need to be investigated when trying to understand individual development across the life course (Bernardi et al., 2018).

For a long time, the CLPM has been the primary tool to analyze these interdependencies. However, due to its strict assumptions, the CLPM might not always be suitable for addressing the sophisticated questions raised by contemporary life course researchers (Allison, 2009; Berry \& Willoughby,
2017; Hamaker et al., 2015). Fortunately, the methodological toolkit of life course researchers has evolved alongside their research questions and many analytic approaches relaxing the strict assumptions of the classical CLPM are readily available. With the RI-CLPM (Hamaker et al., 2015), the ALT-SR (Berry \& Willoughby, 2017; Curran et al., 2014), and the DCSM (Grimm et al., 2012), we have discussed three of these modern tools. It should be noted that none of these models is a Jack-of-all-trades; each comes with its own strengths and weaknesses and one of them might be more appropriate in a given research context than the other. Thus, selecting one model in favor of the other in a given research scenario requires contemporary life course researchers to carefully weigh the benefits and disadvantages of the models against each other to chose the model that best fits their current research question.

Keeping this remark in mind, the models presented here bear the potential to leap forward the understanding of second-order interdependencies across the life course. These models equip life course researchers with the tools to examine a wide variety of vibrant research questions on the interplay between two (or more) variables within persons and the between-person differences in this interplay. Taking temporal interdependence seriously, the presented models enable researchers to investigate truly dynamic aspects of the life course as it is possible to scrutinize the influence of changes in one variable on subsequent change in another and to examine how these changes influence further variables (or changes therein). Thus, life course researchers are equipped to investigate more sophisticated questions and are not restricted to the possibilities of the CLPM, both in terms of theory and analysis. Notably, the models presented here are extraordinarily flexible and can be further extended in various ways. For instance, it is straightforward to implement the models as dyadic models, for instance when studying partner relationships (e.g., Mund et al., 2015). Similarly, it is also possible to analyze selection effects (e.g., 
do RS and/or SE predict childbearing?) when a certain life event occurs during the course of a study. The presented models further allow to examine how (psychological) characteristics and the interplay between multiple constructs, and hence a developmental system, adapt to these life events.

To summarize, the novel statistical approaches presented in this article allow researchers to investigate a wide variety of innovative and complex questions that might truly advance the understanding of individual development across the life course. We hope that these tools will prove useful for the analysis of data already available and for designing new studies aimed at investigating the life course alike. We believe that a more routine use of these models can also inspire new theoretical accounts of individual development across the life course.

\section{References}

Allison, P. D. (2009). Fixed effects regression models. Thousand Oaks: SAGE.

Bernardi, L., Huinink, J., \& Settersten, R. (2018). The life course cube: A tool for studying lives. Advances in Life Course Research, THIS ISSUE, INSERT PAGE NUMBERS WHEN DETERMINED.

Berry, D. \& Willoughby, M. T. (2017). On the practical interpretability of cross-lagged panel models: Rethinking a developmental workhorse. Child Development, 88, 11861206. doi:10.1111/cdev. 12660

Biesanz, J. C. (2012). Autoregressive longitudinal models. In R. H. Hoyle (Ed.), Handbook of structural equation modeling (pp. 459-471). New York: Guilford Press.

Bollen, K. A. \& Zimmer, C. (2010). An overview of the autoregressive latent trajectory (ALT) model. In K. van Montfort, J. Oud, \& A. Satorra (Eds.), Longitudinal research with latent variables (pp. 153-176). New York: Springer.
Brüderl, J., Kratz, F., \& Bauer, G. (2018). Life course research with panel data: An exemplary analysis on the reproduction of social inequality. Advances in Life Course Research, THIS ISSUE, INSERT PAGE NUMBERS WHEN DETERMINED.

Castro-Schilo, L. \& Grimm, K. J. (2018). Using residualized change versus difference scores for longitudinal research. Journal of Social and Personal Relationships, 35, 3258. doi:10.1177/0265407517718387

Curran, P. J. (2003). Have multilevel models been structural equation models all along? Multivariate Behavioral Research, 38, 529-569. doi:10.1207/s15327906mbr3804_5

Curran, P. J. \& Bollen, K. A. (2001). The best of both worlds: Combining autoregressive and latent curve models. In L. M. Collins \& A. G. Sayer (Eds.), New methods for the analysis of change (pp. 105-136). Washington, D. C.: American Psychological Association.

Curran, P. J., Howard, A. L., Bainter, S. A., Lane, S. T., \& McGinley, J. S. (2014). The separation of between-person and within-person components of individual change over time: A latent curve model with structured residuals. Journal of Consulting and Clinical Psychology, 82, 879-894. doi:10 . 1037 / a0035297

Curran, P. J., Obeidat, K., \& Losardo, D. (2010). Twelve frequently asked questions about growth curve modeling. Journal of Cognition and Development, 11, 121-136.

Erol, R. Y. \& Orth, U. (2014). Development of self-esteem and relationship satisfaction in couples: Two longitudinal studies. Developmental Psychology, 50, 2291-2303. doi:10. 1037/a0037370

Grimm, K. J., An, Y., McArdle, J. J., Zonderman, A. B., \& Resnick, S. M. (2012). Recent changes leading to subsequent changes: Extensions of multivariate latent difference score models. Structural Equation Model- 
ing, 19, 268-292. doi:10.1080/10705511. 2012.659627

Grimm, K. J., Ram, N., \& Estabrook, R. (2017). Growth modeling: Structural equation and multilevel modeling approaches. New York: Guilford.

Hamagami, F. \& McArdle, J. J. (2001). Advanced studies of individual differences linear dynamic models for longitudinal data analysis. In G. A. Marcoulides \& R. E. Schumacker (Eds.), New developments and techniques in Structural Equation Modeling (pp. 203246). Mahwah, NJ: Lawrence Erlbaum.

Hamaker, E. L. (2005). Conditions for the equivalence of the autoregressive latent trajectory model and a latent growth curve with autoregressive disturbances. Sociological Methods and Research. doi:10.1177/ 0049124104270220

Hamaker, E. L., Kuiper, R. M., \& Grasman, R. P. P. P. (2015). A critique of the crosslagged panel model. Psychological Methods, 20, 102-116. doi:10.1037/a0038889

Hertzog, C. \& Nesselroade, J. R. (2003). Assessing psychological change in adulthood: An overview of methodological issues. Psychology and Aging, 18, 639-657. doi:10.1037/ 0882-7974.18.4.639

Hox, J. J. (2010). Multilevel analysis: Techniques and applications (2nd ed.). New York: Routledge.

Huinink, J., Brüderl, J., Nauck, B., Walper, S., Castiglioni, L., \& Feldhaus, M. (2011). Panel analysis of intimate relationships and family dynamics (pairfam): Conceptual framework and design. Zeitschrift für Familienforschung, 23, 77-101.

Jongerling, J. \& Hamaker, E. L. (2011). On the trajectories of the predetermined ALT model: What are we really modeling? Structural Equation Modeling, 18, 370-382. doi:10 . 1080/10705511.2011.582004
Kline, R. B. (2011). Principles and practice of structural equation modeling (3rd ed.). New York: Guilford Press.

McArdle, J. J. (2009). Latent variable modeling of differences and changes with longitudinal data. Annual Review of Psychology, 60, 577605. doi:10.1146/annurev.psych.60.110707. 163612

McArdle, J. J. \& Hamagami, F. (2001). Latent difference score structural models for linear dynamic analyses with incomplete longitudinal data. In L. M. Collins \& A. G. Sayer (Eds.), New methods for the analysis of change (pp. 137-175). Washington, DC: American Psychological Association.

Mund, M., Finn, C., Hagemeyer, B., Zimmermann, J., \& Neyer, F. J. (2015). The dynamics of self-esteem in partner relationships. European Journal of Personality, 29, 235-249. doi:10.1002/per.1984

Mund, M., Zimmermann, J., \& Neyer, F. J. (2018). Personality development in adulthood. In V. Zeigler-Hill \& T. K. Shackelford (Eds.), The SAGE handbook of personality and individual differences (Vol. 2: Origins of personality and individual differences, pp. 260-277). Los Angeles: Sage.

Muthén, L. K. \& Muthén, B. O. (2017). Mplus user's guide (8th ed.). Los Angeles: Muthén \& Muthén.

Nestler, S., Grimm, K. J., \& Schönbrodt, F. D. (2015). The social consequences and mechanisms of personality: How to analyse longitudinal data from individual, dyadic, roundrobin and network designs. European Journal of Personality, 29, 272-295. doi:10 . 1002/per.1997

Orth, U., Erol, R. Y., \& Luciano, E. C. (2018). Developmnt of self-esteem from age 4 to 94 years: A meta-analysis of longitudinal studies. Psychological Bulletin, 144, 1045-1080. doi:10.1037/bul0000161

Orth, U., Robins, R. W., \& Widaman, K. F. (2012). Life-span development of self-esteem and its 
effects on important life outcomes. Journal of Personality and Social Psychology, 102, 1271-1288. doi:10.1037/a0025558

Ram, N. \& Grimm, K. J. (2007). Using simple and complex growth models to articulate developmental change: Matching theory to method. International Journal of Behavioral Development, 31, 303-316. doi:10 . 1177 / 0165025407077751

Rosseel, Y. (2012). lavaan: An R package for structural equation modeling. Journal of Statistical Software, 48, 1-36. Retrieved from http: //www.jstatsoft.org/v48/i02/paper

Snijders, T. A. B. \& Bosker, R. J. (2012). Multilevel analysis: An introduction to basic and advanced multilevel modeling (2nd ed.). London: SAGE.

Steyer, R., Eid, M., \& Schwenkmezger, P. (1997). Modeling true intraindividual change: True change as a latent variable. Methods of Psychological Research Online, 2, 21-33. Retrieved from http : / / www . dgps . de / fachgruppen/methoden/mpr-online/issue2/ art2/steyer.pdf

Trzesniewski, K. H., Donnellan, M. B., \& Robins, R. W. (2003). Stability of self-esteem across the life span. Journal of Personality and Social Psychology, 84, 205-220. doi:10.1037/ 0022-3514.84.1.205

VanderWeele, T. J., Hawkley, L. C., Thisted, R. A., \& Cacioppo, J. T. (2011). A marginal structural model analysis for loneliness: Implications for intervention trials and clinical practice. Journal of Consulting and Clinical Psychology, 79, 225-235.

Voelkle, M. C. (2008). Reconsidering the use of autoregressive latent trajectory (ALT) models. Multivariate Behavioral Research, 43, 564-591. doi:10.1080/00273170802490665

Voelkle, M. C. \& Oud, J. H. L. (2013). Continuous time modelling with individually varying time intervals for oscillating and non-oscillating proscesses. British Journal of Mathematical and Statistical Psychology,
66, 103-126. doi:10.1111/j . 2044 - 8317. 2012.02043.x

Voelkle, M. C. \& Oud, J. H. L. (2015). Relating latent change score and continuous time models. Structural Equation Modeling, 22, 366381. doi:10.1080/10705511.2014.935918

Voelkle, M. C., Oud, J. H. L., Davidov, E., \& Schmidt, P. (2012). An SEM approach to continuous time modeling of panel data: Relating authoritarianism and anomia. Psychological Methods, 17, 176-192. doi:10.1037/ a0027543 
Appendix

In this Appendix, we present the formulas of the models described in the main part of the manuscript. We start with the Cross-Lagged Panel Model (CLPM). This is followed by the RandomIntercept-CLPM (RI-CLPM), the Autoregressive Latent Trajectory Model with Structured Residuals (ALT-SR), and the Dual Change Score Model (DCSM).

Cross-Lagged Panel Model. The CLPM allows to examine rank-order changes in two variables and time-lagged associations between two variables. The CLPM is based on a true-score formulation of the observed variables $x$ and $y$ of person $i$ (see Biesanz, 2012; Grimm et al., 2017)

$$
\begin{aligned}
& x_{t i}=l x_{t i}+u_{t i} \\
& y_{t i}=l y_{t i}+v_{t i},
\end{aligned}
$$

where $x_{t i}$ and $y_{i t}$ are the observed values of person $i$ at time $t$ (for all $t>0$; e.g., self-esteem and relationship satisfaction at the third time point), $l x_{t i}$ and $l y_{i t}$ are the latent true scores and $u_{t i}$ and $v_{t i}$ are the residuals of the observed variables. Given these definitions, the CLPM equations for person $i$ are

$$
\begin{aligned}
& x_{t i}=g_{t 0}+a_{t-1,1} l x_{t-1, i}+c_{t-1,2} l y_{t-1, i}+\operatorname{lr} x_{t i}+u_{t i} \\
& y_{t i}=b_{t 0}+a_{t-1,2} l y_{t-1, i}+c_{t-1,1} l x_{t-1, i}+\operatorname{lr} y_{t i}+v_{t i},
\end{aligned}
$$

where $\operatorname{lr} x_{t i}$ and $\operatorname{lr} y_{t i}$ are time point specific residual variables of the true scores. $g_{0 t}$ and $b_{0 t}$ are time point specific intercepts. $a_{t-1,1}$ and $a_{t-1,2}$ are autoregressive parameters that can be interpreted in terms of the rank-order stability over time. Finally, $c_{t-1,1}$ and $c_{t-1,2}$ are cross-lag parameters describing the association between scores of a variable at the previous time point and subsequent scores (measured at occasion $t, t>0$ ) in the other variable. All other terms are defined as in Equation 1
When estimating a CLPM, it is common to constrain the autoregressive and cross-lagged parameters to the same values across measurement waves. In this case, the time index would drop from the coefficients. Furthermore, it is typically assumed that the residual scores of the observed variables have equal variance across time points, that the variance of the latent residual scores except the first one (e.g., $\operatorname{lr} x_{t i}, \operatorname{lr} y_{t i}$ ) are time-invariant, and that the correlation between these terms is time- invariant. For the residual scores of the initial time point, finally, one typically estimates specific variance and correlation parameters.

\section{Random-Intercept Cross-Lagged Panel} Model. The RI-CLPM extends the CLPM by defining latent intercept factors for each of the two measures:

$x_{t i}=\left(g i_{0}+i x_{i}\right)+a_{t-1,1} l x_{t-1, i}+c_{t-1,2} l y_{t-1, i}+\operatorname{lr} x_{t i}+u_{t i}$
$y_{t i}=\left(b i_{0}+i y_{i}\right)+a_{t-1,2} l y_{t-1, i}+c_{t-1,1} l x_{t-1, i}+\operatorname{lr} x_{t i}+v_{t i}$,

where $i x_{i}$ and $i y_{i}$ denote the deviation of person $i$ from the mean values in the latent intercept factor for the repeatedly measured variable $x$ (e.g., self-esteem) and $y$ (e.g., relationship satisfaction), and $g i_{0}$ and $b i_{0}$ are the means of these intercept factors. All other variables are defined in the same way as in the standard CLPM.

In contrast to the CLPM, the RI-CLPM does not include the measurement error terms of the observed indicators (e.g., the variance terms of these residual scores are constrained to be zero). Furthermore, when the means of the intercept factors are included in Equation 3, time point specific intercepts have to constrained to be zero. The model also assumes that the two intercept factors are correlated and that the latent residual scores (e.g., $\operatorname{lr} x_{t i}, \operatorname{lr} y_{t i}$ ) are correlated variables. Finally, the variance of the latent residuals is constrained to the same value across all time point except the first one. Also, and similar to the CLPM, most researchers constrain the autoregressive parameters and the cross-lagged parameters to the same value 
across measurement waves. In this case, the time index would again drop from the coefficients.

\section{Autoregressive Latent Trajectory Model} with Structured Residuals. The ALT-SR extends the CLPM by including a latent intercept factor and a latent slope factor capturing linear change for each of the two involved variables. The model is given by:

$x_{i, t}=\left(g i_{0}+i x_{i}\right)+\lambda_{i}\left(g s_{0}+s x_{i}\right)+a_{t-1,1} l x_{t-1, i}+c_{t-1,2} l y_{t-1}$

$$
\begin{aligned}
& x_{i, t}=l x_{i, t-1}+d x_{i, t}+l u_{i, t}+u_{i, t} \\
& y_{i, t}=l y_{i, t-1}+d y_{i, t}+l v_{i, t}+v_{i, t},
\end{aligned}
$$

where $d x_{i, t}$ and $d x_{i, t}$ denote the values of person $i$ in the latent change score variable for $x$ and $y$ at time point $t$, respectively. All other terms are defined as in the standard CLPM. The DCSM further assumes that the latent change scores occurring in Equation 5 are functions of a constant change com$y_{i, t}=\left(b i_{0}+i y_{i}\right)+\lambda_{i}\left(b s_{0}+s y_{i}\right)+a_{t-1,2} l y_{t-1, i}+c_{t-1,1} l x_{t-Y, i}$ Yous

Here, $s x_{i}$ and $s y_{i}$ denote the slope factors and $g s_{0}$ and $b s_{0}$ are the means of these factors across persons. $\lambda_{i}$ is the loading of the slope at time point $t$. All other variables are defined in the same way as in the CLPM or in the RI-CLPM.

Similar to the RI-CLPM, the ALT-SR does not include the measurement error terms of the observed indicators. The time point specific intercepts are also constrained to be zero. Furthermore, the model estimates all correlations between the intercept factors, between the slope factors, and between the intercept and the slope factors. Also, the correlations between the latent residual scores of the two variables at each time point are freely estimated. However, the latter variables are assumed to be uncorrelated with the intercept and slope factors, respectively. Finally, equality constraints might be imposed for the autoregressive parameters, the cross-lag parameters, and the correlations between the latent residual scores.

Dual Change Score Model. The DCSM also uses a true score formulation to model the longitudinal trajectories and dynamics of the involved variables. However, and in contrast to the other three models, the DCSM models changes between measurement occasions (and not growth). Therefore, changes are explicitly incorporated into the model equations. For two variables, the basic equations are

$$
\begin{aligned}
d x_{i, t} & =g s_{0}+s x_{i}+a_{1} l x_{i, t-1}+c_{2} l y_{i, t-1} \\
d y_{i, t} & =b s_{0}+s y_{i}+a_{2} l y_{i, t-1}+c_{1} l x_{i, t-1} .
\end{aligned}
$$

Here, $s x_{i}$ and $s y_{i}$ denotes person $i$ 's values in the constant change component for variable $x$ and $y$, respectively, and $g s_{0}$ and $b s_{0}$ denote the means of these factors across persons. $a_{1}$ and $a_{2}$ denote the influence of the variable at the previous time point on the same variable at time $t$, and $c_{1}$ and $c_{2}$ reflect the influence of the other variable at the previous time point $t-1$. Per assumption, both types of parameters are assumed to be time-invariant. Finally, we note that as the change score variables are latent variables, the model should also contain residuals for these change scores. However, it is assumed that the variance of these residuals is zero. We therefore decided to omit these terms in Equation 6.

When estimating the DCSM it is assumed that the intercepts of the observed indicators and the means of the true scores, except the true scores for the first time point, are zero. The means of the constant change component is also not set to zero but is estimated. Furthermore, the variance terms of the latent residuals except the first one are set to zero. This allows to estimate all correlations between these two true score variables and the latent factors representing the constant change component. All other variables are assumed to be uncorrelated with these latent variables. Finally, 
the variance terms of the residual scores of the ob- estimation. served indicators are set to the same value during 